\title{
compression de milieux granulaires modèles à deux dimensions
}

\section{compression of model two dimensional granular media}

\author{
Équipe de physique des matériaux désordonnés \\ T. TRAVERS, M. AMMI, D. BIDEAU, \\ A. GERVOIS*, J. LEMAITRE, J.C. MESSAGER ET J.P. TROADEC \\ Groupe de Physique Cristalline."
}

\section{Résumé}

Nous présentons une synthèse de travaux menés par notre équipe sur des empilements $2 d$ de cylindres soumis à une compression verticale œdométrique.

Nous avons étudié les effets du désordre géométrique et du désordre de composition (mélange de grains durs et mous) en utilisant en particulier des observations en photoélasticité. Ces expériences font apparaître deux régimes en fonction de la force appliquée : un régime de consolidation et un régime consolidé : dans chacun d'eux la relation force macroscopique-déformation peut être exprimée par une loi en puissance dont l'exposant est très différent de l'exposant microscopique de Hertz et dépend beaucoup de la qualité des contacts entre grains.

Nous avons également fait l'analyse critique de ces expériences en estimant l'importance des effets de taille finie et ceux des parois. Le rôle des frottements grain-paroi est discuté et le rapport force transmise/force appliquée a été mesuré. Nous avons testé l'influence de la taille de l'échantillon en augmentant la largeur, la hauteur ou les deux simultanément. Nous mettons ainsi en évidence le rôle d'une longueur caractéristique supérieure à la taille des grains.

\footnotetext{
Abstract

We present a synthesis of some experiments realized by our group on $2 d$ packings of cylinders submitted to a vertical cedometric stress.

We have studied the effects of geometrical disorder and composition disorder (mixtures of soft and hard grains); results are often supported by photoelacticity observations. From these experiments, two regimes clearly appear, a consolidation and a consolidated ones, depending on the force which is applied. In both cases, the strain-stress relation may be fitted with a power law, the exponent of which is different from the microscopical Hertz exponent and strongly depends on the quality of the contacts between grains.

We have also performed the critical analysis of these studies by estimating the role of the walls and of the finite size effects. The influence of the frictions of the grains against the walls is discussed and the ratio of the transmitted to the applied force has been measured. We have tested the role of the size of the samples by increasing their width, height or both simultaneously. We thus put into evidence the existence of a characteristic length, greater than the grain size.
}

\footnotetext{
- Adresse permanente : Service de Physique Théorique, C.E.N. Saclay, 91191 Gif sur Yvette Cedex,

* Campus de Beaulieu, Université de Rennes I, 35042 Rennes Cedex.
} 


\section{INTRODUCTION}

Les milieux granulaires non consolidés - qui constituent un matériau de base pour le génie civil - sont hétérogènes à une échelle macroscopique. En effet, il y coexiste généralement :

- un désordre de position des éléments constitutifs,

- un désordre de composition, lié à l'existence de plusieurs phases dont le contraste des propriétés physiques ou physicochimiques peut être important,

- des interfaces de contact dont le rôle est souvent primordial.

Leurs propriétés sont difficiles à analyser, étant donné le grand nombre de paramètres dont elles dépendent : taille et nature du * récipient ", morphologie des grains (forme, taille et dispersion de taille), nature des conctacts entre grains... L'importance relative de ces paramètres peut varier suivant \& l'histoire " du milieu, en particulier au niveau du mode de construction et des contraintes mécaniques subies.

La nécessité de modèles simplifiés se fait alors sentir, modèles qui seront d'autant mieux adaptés qu'ils permettent de sérier l'influence d'un ou plusieurs de ces paramètres sur les propriétés du milieu. Ceci explique le succès en mécanique des sols des modèles d'empilements $3 \mathrm{~d}$ de sphères ou $2 \mathrm{~d}$ de cylindres parallèles (modèle de SCHNEEBELI, 1956), modèles présentant éventuellement une distribution de taille des « grains $x$.

Lorsqu'un matériau granulaire est soumis à une force extérieure, cette force est transmise par l'intermédiaire des contacts entre les grains. Pour deux grains sphériques, la force $f$, appliquée au contact et supposée radiale, est reliée à la diminution $\Delta \mathrm{x}$ de la distance entre les centres par la loi de Hertz

$$
f \sim(\Delta \mathrm{x})^{\mu}
$$

avec $\mu=1,5$. Cette loi est également assez bien vérifiée dans le cas de grains non sphériques, même si la force comporte une composante tangentielle.

Au niveau macroscopique, les matériaux granulaires, lorsqu'ils sont soumis à une compression œedométrique, ont également une caractéristique contraintedéformation le plus souvent non linéraire (FEDA 1982)

$$
F=F_{0}\left(\Delta h / h_{0}\right)^{m}
$$

où $\Delta h$ est l'aplatissement global de l'échantillon sous l'effet de la force macroscopique $F, m$ l'exposant macroscopique, $h_{0}$ la hauteur initiale de l'empilement et $F_{\text {o }}$ un préfacteur. Généralement, l'exposant macroscopique $\mathrm{m}$ est différent de l'exposant microscopique $\mu$; il dépend de la nature du matériau constitutif, de l'état de surface des grains, de l'histoire du milieu, etc. ; il n'est pas non plus constant sur la totalité de l'échelle de force explorée. On observe en effet 3 régimes quand la force augmente :

i) à * faible force ", la plus grande partie de la déformation est due à des déplacements locaux des grains. La gamme de force dans laquelle ce comportement est observé dépend de la géométrie de l'empilement, des caractéristiques mécaniques des grains ainsi que des frottements entre grains :

ii) dans la gamme de « force intermédiaire », qui correspond souvent au cas expérimental (en particulier quand l'empilement a été comprimé une première fois) la plus grande partie de la déformation au niveau local est due à la compression de type Hertz aux contacts entre grains, mais tous les contacts possibles ne sont pas actifs (par contact actif, nous entendons contact qui transmet une force) ; le nombre de contacts actifs augmente avec la force extérieure.

iii) à * force élevée », tous les contacts sont actifs, ou du moins le nombre de contacts actifs ne varie pratiquement plus : le comportement macroscopique est donné par la loi de Hertz. Une telle situation est tout à fait exceptionnelle car elle implique de telles forces, que pour deux grains individuels comme pour l'empilement total, la réponse n'est alors plus élastique (domaine de plasticité, rupture, etc. (HARTMANN 1964))

Ces régimes ont été simulés sur un réseau régulier de " diodes Zener symétriques $»$, avec une distribution des tensions de seuil des diodes (ROUX et HERRMANN 1987). Les fluctuations des tensions de seuil correspondent aux variations du nombre de contacts actifs, la caractéristique microscopique courant-tension au-dessus du seuil correspond à la loi microscopique de Hertz quand le contact est actif. L'exposant calculé dans la zone où les diodes ne sont pas toutes actives est différent de l'exposant microscopique, comme dans nos études mécaniques.

Nous avons étudié, dans le cas de compression œedométrique, la loi contrainte-déformation sur l'empilement modèle $2 \mathrm{~d}$ (c'est-à-dire à 2 dimensions) de Schneebeli. Ce modèle présente certains avantages:

- les grains sont de forme simple ;

- il est possible de déterminer la géométrie de l'empilement à partir d'observations directes :

- en choisissant convenablement le matériau constitutif des cylindres (plexiglas par exemple), on peut visualiser les contraintes intergranulaires par photoélasticité.

Les empilements étudiés sont constitués de cylindres " identiques » arrangés de façon à former un réseau triangulaire « régulier *. En réalité ces cylindres sont caractérisés par une distribution de diamètre effectivement étroite du point de vue géométrique, mais qui, comme nous le verrons, peut être importante pour les propriétés mécaniques.

Après avoir décrit le dispositif expérimental (section 2), nous proposons une analyse géométrique des empilements (section 3). La section 4 est consacrée à l'étude de l'influence, sur la réponse mécanique, du désordre géométrique causé par la mauvaise définition du diamètre des cylindres ; nous nous appuyons sur la visualisation des contraintes par photoélasticité pour rendre compte qualitativement des résultats obtenus. Dans la section 5 , nous considérons le cas du désordre de composition créé en mélangeant deux types de cylindres de propriétés mécaniques différentes. Dans la section 6 , nous étudions par différentes méthodes l'influence de la taille des échantillons sur la réponse mécanique ; 
nous mettons ainsi en évidence l'existence d'une longueur caractéristique que nous discutons dans la dernière section.

\section{DISPOSITIF EXPÉRIMENTAL}

La plupart des expériences ont été effectuées sur des empilements de cylindres de plexiglas de $2,5 \mathrm{~cm}$ de longueur. Ces cylindres présentent des défauts géométriques (fluctuations de diamètre, ellipticité, courbure) ; leur diamètre peut être estimé à $4,0 \pm 0,1 \mathrm{~mm}$. Dans l'empilement, deux cylindres ne sont pas en contact tout au long de leur génératrice commune (ils sont faiblement croisés et ne sont pas tout à fait rectilignes) ; la loi de Hertz s'applique alors au niveau microscopique avec un exposant $\mu$ de l'ordre de 1,5 ; expérimentalement, nous avons mesuré $\mu=1,8 \pm 0,1$. Par ailleurs, en particulier pour l'étude du désordre de composition, nous avons également utilisé des cylindres de caoutchouc de même diamètre que les cylindres de plexiglas, mais qui peuvent être considérés comme géométriquement mieux définis, étant donnée l'amplitude de leur déformation.

L'empilement le plus grand est constitué de 48 rangées comportant alternativement 44 et 45 cylindres formant un réseau triangulaire ; il est construit dans un cadre rigide, puis placé dans une machine d'essais universelle "INSTRON 1175 », comme schématisé en figure 1. Un déplacement vertical $\Delta h$ est imposé sur le plateau inférieur et nous mesurons la force de compression correspondante $\mathrm{F}$. Les expériences sont effectuées à vitesse lente $(0,2 \mathrm{~mm} / \mathrm{mn})$; ainsi, comme nous lavons vérifié, les phénomènes observés sont indépendants de la vitesse. Plusieurs cycles de pression sont exécutés, jusqu'à ce que les résultats soient stabilisés et reproductibles ( 3 cycles pour un réseau régulier, à peu près 40 cycles pour un empilement désordonné). La gamme de force explorée (excepté dans la section 6-4) est $0-2000 \mathrm{~N}$.

La procédure expérimentale décrite ci-dessus appelle quelques commentaires. Au premier cycle, il y a beaucoup de réorganisations locales qui ccnduisent à des modifications géométriques irréversibles, par glissements ou rotations ; beaucoup de voûtes disparaissent par exemple. Ces réorganisations sont très visibles

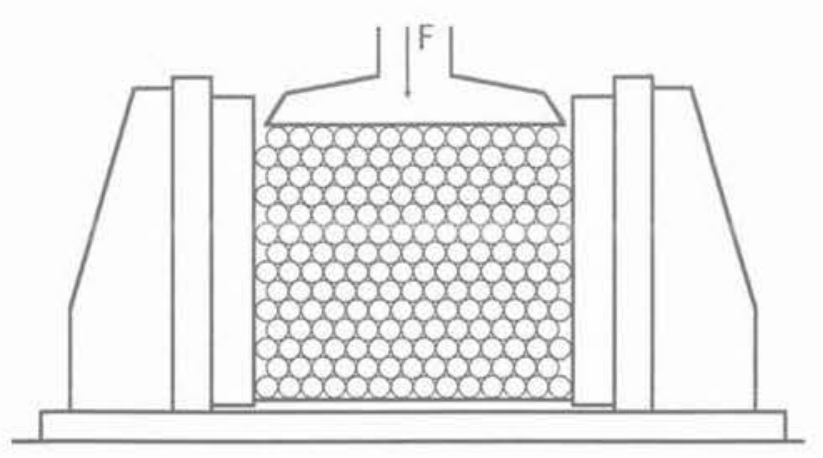

Fig. 1. - Schéma du dispositif.

Fig. 1. - Experimental apparatus scheme. dans les empilements désordonnés où des amas constitués de plusieurs grains peuvent glisser de façon collective ; ceci est également vrai dans nos empilements ordonnés, mais les déplacements sont moins importants. Les quantités mesurées dépendent de la disposition géométrique des grains au repos et sont différentes d'un échantillon à l'autre. Ainsi le premier cycle n'est pas du tout représentatif pour notre étude et ne doit pas être pris en considération pour la détermination de la loi contrainte-déformation, même si les déformations qu'il provoque peuvent influencer la réponse ultérieure. La place des grains reste pratiquement fixe au cours des cycles suivants. A faible force, ils peuvent encore se réarranger un peu ; quelques contacts disparaissent et d'autres se créent, mais les changements sont locaux et en partie réversibles. Ceci correspond à la zone (i) dans l'introduction, et peut être considéré comme une phase de a consolidation ». Quand la pression verticale augmente, les grains ne bougent pratiquement plus et les déformations sont essentiellement de type Hertz aux contacts (zone (ii) de l'introduction ou phase « consolidée ).

Nous avons eu à résoudre deux problèmes techniques. Tout d'abord, le profil supérieur de l'empilement est irrégulier à cause des hétérogénéités ; il n'est donc pas aisé de définir le zéro de déplacement : le définit-on à partir du premier grain en contact avec le piston, ou à partir de la position moyenne des grains qui sont dans la première couche? Comme les déplacements sont très faibles, une bonne détermination de ce zéro conditionne l'obtention d'une valeur précise de l'exposant $\mathrm{m}$. Expérimentalement, il est plus efficace de définir le zéro à partir de la force ; dans notre cas, ce zéro est déterminé avec une précision de $0,2 \mathrm{~N}$.

Le second problème concerne la loi force-déformation: elle n'est pas la même suivant que la force augmente ou diminue. Des hystérésis existent encore après plusieurs cycles de pression, mais ils sont rapidement stabilisés et reproductibles. Les mesures ont été effectuées à force croissante ; dans ces conditions, le coefficient de pression latérale $\mathrm{K}_{\mathrm{o}}$ peut être considéré comme constant (voir section 6-2).

\section{ANALYSE GÉOMÉTRIQUE}

Dans un premier temps, nous supposons que l'empilement est très grand. Quand aucune force macroscopique n'est appliquée, sa structure présente quelques particularités qui conditionnent en partie son comportement ultérieur. Dans notre cas, il est construit de façon non collective, proche d'une construction grain par grain, et sous gravité. Chaque nouveau grain est en équilibre s'il a deux contacts avec l'empilement, et on peut penser que la distribution de taille des grains est telle que la probabilité d'avoir un troisième contact est nulle. La coordinance moyenne (nombre moyen de contacts par grain) est alors égale à 4. Mais les contacts ne sont pas distribués de façon égale suivant les 3 directions possibles. En utilisant un argument proche de celui de ROUX, STAUFFER et HERRMANN (1987), nous considérons que chaque grain a un contact suivant la direction $\mathrm{Y}$ (ou Z) (figure 2), avec une probabilité égale d'avoir un deuxième contact suivant la direction Z (ou Y) ou X. La probabilité d'existence 
d'un contact est 0,75 suivant la direction $\mathrm{Y}$ ou $\mathrm{Z}$ et est 0,5 suivant la direction $\mathrm{X}$. La distribution spatiale des contacts est donc anisotrope avant toute application de la pression (ceci est également vrai pour des empilements 3d de grains désordonnés (BIAREZ 1962)).

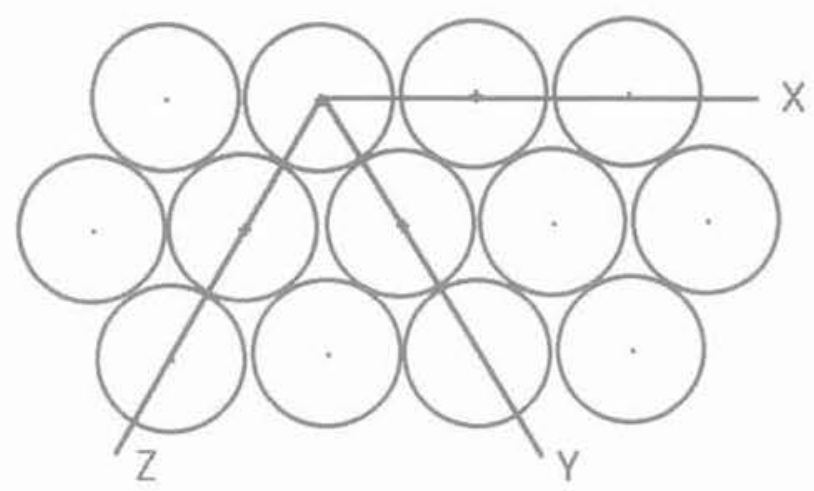

Fig. 2. - Empilement ordonné $2 d$ (réseau triangulaire) Direction des contacts.

Fig. 2. - 2d ordered packings - directions of the contacts.

Si maintenant une force verticale est appliquée, des glissements et des rotations (locaux ou collectifs) se produisent, essentiellement au cours du premier cycle. La compression conduit à une augmentation du nombre de contacts dans les directions $Y$ et $Z$, et à une diminution dans la direction $\mathrm{X}$. L'anisotropie initiale sera accentuée. Nos observations photoélastiques (section 4-2) confirment que les contacts horizontaux transmettent peu les contraintes. Si on ignore les contacts $\mathrm{X}$, le réseau triangulaire peut être remplacé, dans un premier temps, par un réseau losange lavec un angle de $60^{\circ}$ ). Nous insistons sur le fait que l'analyse précédente est seulement vraie en moyenne : la situation est très différente d'un échantillon à l'autre puisque des positions légèrement différentes des grains au repos (avant application de la force) peuvent considérablement modifier la répartition des contraintes quand la force est appliquée.

Les parois horizontales et verticales peuvent jouer un grand rôle dans la définition géométrique de l'empilement : un bord rigide induit, à son voisinage, des modifications de structure de l'échantillon. Dans un système désordonné, la porosité ou la coordinance par exemple sont différentes de celles en volume sur des distances pouvant atteindre plusieurs diamètres de grains (BEN AIM et LE GOFF 1968). Certaines propriétés physiques peuvent s'en trouver fortement perturbées ; c'est le cas, par exemple de la dispersion hydrodynamique, très sensible aux inhomogénéités de porosité (GAUTHIER, BORGOTTI et SADOUDI 1987). Dans notre système ordonné, on considère que ces changements géométriques sont négligeables le long des parois verticales, au moins au-delà de la première colonne de grains. Pour la paroi horizontale inférieure, les grains sont tous, par gravité, en contact avec elle : nous admettons que ceci n'altère pas le comportement mécanique de notre système.
La première application de pression est intéressante pour les modifications géométriques qu'elle implique, car, pour une même pression maximale, l'empilement garde la mémoire de ces modifications durant les cycles de pression ultérieurs (DANTU 1967). Nous avons vu que les grains de la couche supérieure forment une « surface irrégulière n : ceci implique des modifications géométriques à l'échelle de plusieurs rangées quand la pression est appliquée par l'intermédiaire du piston. La structuration est faible dans un empilement ordonné mais très visible dans un échantillon désordonné.

Pour étudier ce problème, nous avons construit un empilement désordonné de grains monotailles $(\Phi=$ $0,4 \mathrm{~cm})$, le désordre étant obtenu en placant quelques grains de plus grand diamètre $(\Phi=1 \mathrm{~cm})$ le long des parois horizontales et verticales du cadre rigide (BIDEAU 1983). Avant que la pression ne soit appliquée, des lignes horizontales ont été tracées sur la section circulaire des cylindres, d'une paroi verticale à l'autre. Durant la première application de la pression, les déformations de ces lignes montrent des réarrangements géométriques forts (glissements, rotations) dans les quatre ou cinq premières couches de l'échantillon. Au-delà de ces rangées, les lignes sont moins déformées, la compression est plus homogène ; elle ne semble pas dépendre de la distance à la paroi supérieure. Ainsi, on peut penser que, d'un point de vue géométrique, l'effet de la paroi active (celle qui applique la pression) n'est pas très différent de celui d'une paroi fixe. Cependant, même dans notre système ordonné, cet effet peut engendrer d'importantes modifications dans la distribution des contacts dans les premières couches et donc conduire à des corrélations « mécaniques " à grande distance.

\section{LOI CONTRAINTE-DÉFORMATION ÉTUDE PHOTOÉLASTIQUE}

Nous étudions, dans la zone de force intermédiaire, de * grands * empilements constitués de 48 rangées de 44 ou 45 cylindres de plexiglas disposés selon le réseau triangulaire régulier. La mauvaise définition des cylindres est à l'origine d'un désordre géométrique faible. mais suffisant pour affecter la loi contraintedéformation. La visualisation des contraintes par photoélasticité permet une approche qualitative du comportement observé,

\subsection{Loi macroscopique contrainte-déformation}

La montée en pression fait apparaître deux régimes : à faible force, nous avons la zone de "consolidation " durant laquelle l'échantillon se met en place grâce à des déplacements locaux des grains, réversibles ou quasi réversibles. A force plus élevée l'échantillon est "consolidé x : la déformation globale résulte de déformations locales de type Hertz et peut être décrite par la relation (2) (figure 3), avec $m \sim 3,9 \pm 0,4$ et $\mathrm{F}_{\circ} \sim 10^{11} \mathrm{~N}$. La variation relative de hauteur de l'empilement est de l'ordre de $1 \%$ pour une force compressive de $2000 \mathrm{~N}$. Nous avons vérifié qu'une telle loi reste valable dans une gamme de force allant jusqu'à $10000 \mathrm{~N}$. 


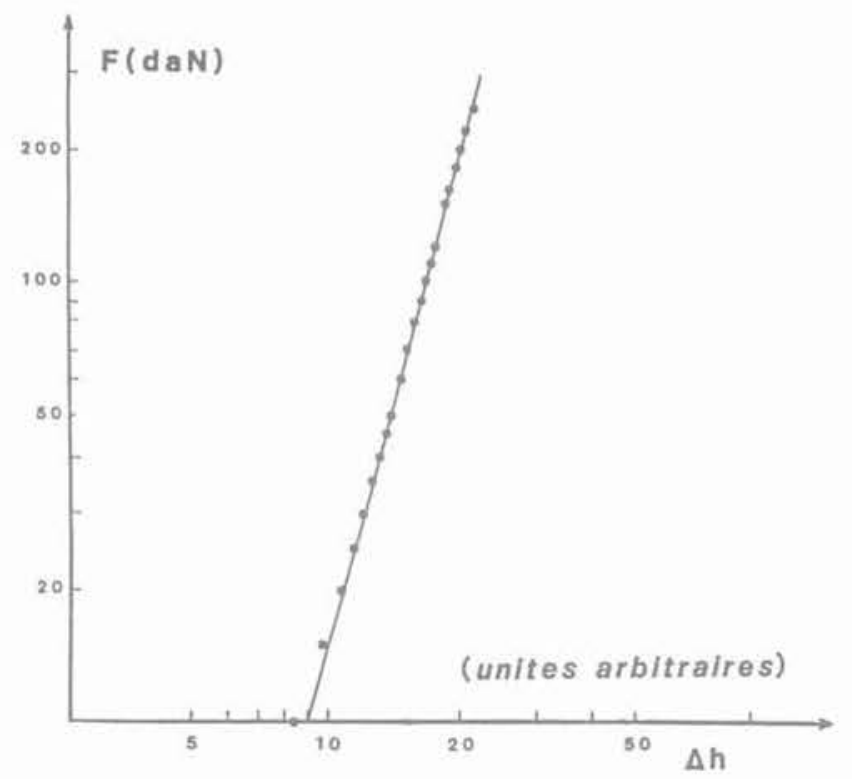

Fig. 3. - Loi force-déformation (en échelle log-log) pour un échantillon de $48 \times 44$ cylindres de plexiglas. Fig. 3. - Stress-strain law (in log-log scale) for a sample made of $48 \times 44$ plexiglass cylinders.

La différence entre la valeur de l'exposant macroscopique $\mathrm{m}(3,9)$ et celle de l'exposant microscopique de Hertz $(1,5)$ peut être attribuée aux hétérogénéités locales créées par les imperfections géométriques des grains, comme le montre la comparaison des résultats obtenus sur des échantillons constitués de matériaux différents. Dans le cas présent, l'échelle des imperfections géométriques, de l'ordre de $100 \mu$, est supérieure à celle de déformations locales de type Hertz, de lordre de $40 \mu$ pour une force de $2000 \mathrm{~N}$; un deuxième lot de cylindres de plexiglas, ayant un meilleur état de surface, a donné $m=3,4 \pm 0,4$; avec un empilement de cylindres d'acier beaucoup mieux calibrés, nous obtenons un exposant $(2,2)$ beaucoup plus proche de l'exposant de Hertz; avec des cylindres de caoutchouc, pour lesquels la déformation est beaucoup plus grande que la taille des inhomégénéités des grains, l'exposant mesuré est compatible avec l'exposant de Hertz (TRAVERS et al. 1986). Les imperfections conduisent à une répartition inhomogène des contacts, donc des contraintes intergranulaires, comme le montrent les expériences de photoélasticité que nous allons maintenant décrire.

\section{2. Étude photoélastique}

Cette étude porte sur des échantillons soumis à une compression telle qu'ils sont dans le régime « consolidé ». L'empilement est placé entre deux polariseurs croisés: les grains très contraints apparaissent alors éclairés, tandis que les grains peu ou pas contraints restent sombres (figure 4). Comme l'avaient observé DANTU (1957) et DRESCHER et de JOSSELIN de JONG (1972), la répartition spatiale des forces intergranulaires n'est pas homogène à l'échelle du grain : les grains les plus contraints génèrent un réseau irrégulier, * le réseau des fortes contraintes ", plus ou moins connecté selon la valeur de la force appliquée. Environ $15 \%$ des contacts sont fortement éclairés, et 20 à $30 \%$ sont moins fortement éclairés. Si on admet que peu de modifications géométriques ont lieu lorsqu'on augmente la pression, on peut penser que les contacts très éclairés sont présents et transmettent la contrainte dès le début, les contacts moins éclairés ne devenant actifs que lorsque la pression augmente. Très peu de contacts horizontaux sont actifs. L'existence du réseau des fortes contraintes, qui transmet la plus grande partie de la force, implique que la déformation macroscopique fasse intervenir des phénomènes à une échelle nettement plus grande que celle du grain : les lois locales sont alors insuffisantes pour décrire le comportement global du système.

L'observation du réseau des fortes contraintes est un bon test de l'homogénéité macroscopique de l'échantillon soumis à une sollicitation mécanique. En effet, une importante question (et qui sera développée plus loin) est de savoir si une des parois a un rôle prédominant quand la force augmente. On peut par exemple penser qu'un grain de la rangée supérieure, s'il est plus haut que les autres, serait le premier à être contraint et pourrait donc générer des lignes de plus fortes contraintes. Celles-ci se développeraient alors selon les directions Z et $Y$ (figure 2) et pourraient se propager loin dans l'empilement, avec parfois un faible nombre de grains contraints à l'intérieur de l'angle correspondant (un cône à 3d). Cet « effet de cône » est lié à la nature vectorielle de la force. Si une force verticale est appliquée sur un grain, elle ne pourra se propager que dans le cône, les contacts horizontaux étant inefficaces.

En fait, il ne semble pas que les grains de la couche supérieure jouent un rôle vraiment particulier : les premiers bras éclairés ne se développent pas à des endroits privilégiés de l'échantillon. En outre, nous avons observé des « effets de cône " partant de grains, qui à première vue sont situés aléatoirement dans lempilement. A cause du caractère vectoriel des forces, les chaînons éclairés forment des bras rectilignes qui se « propagent " aussi loin que le permettent les défauts géométriques de l'empilement ; ces lignes suivent souvent les génératrices des cônes et ainsi nous pouvons considérer que le réseau observé est la superposition de nombreux * cônes * individuels. L'angle d'ouverture de ces cônes (ou la direction des lignes du réseau) dépend de la géométrie de l'empilement. Par exemple, si nous tournons nos empilements * ordonnés de $90^{\circ}$, il n'y a plus de contacts horizontaux. Cette fois les lignes de force utilisent les trois directions possibles (figure 5).

Deux sortes de désordre (ou d'ordre) peuvent donc être importantes pour expliquer ces corrélations à longue portée pour la transmission de la force :

- le désordre de " contact $»$.

- le désordre d'" orientation ».

Dans les empilements ordonnés tels que les nôtres, la force se transmet suivant les directions préférentielles du réseau, si du moins une absence de contact ne bloque pas cette transmission. Lorsque les systèmes sont un peu plus désordonnés, la force peut néanmoins être transmise à distance suivant une direction donnée, 

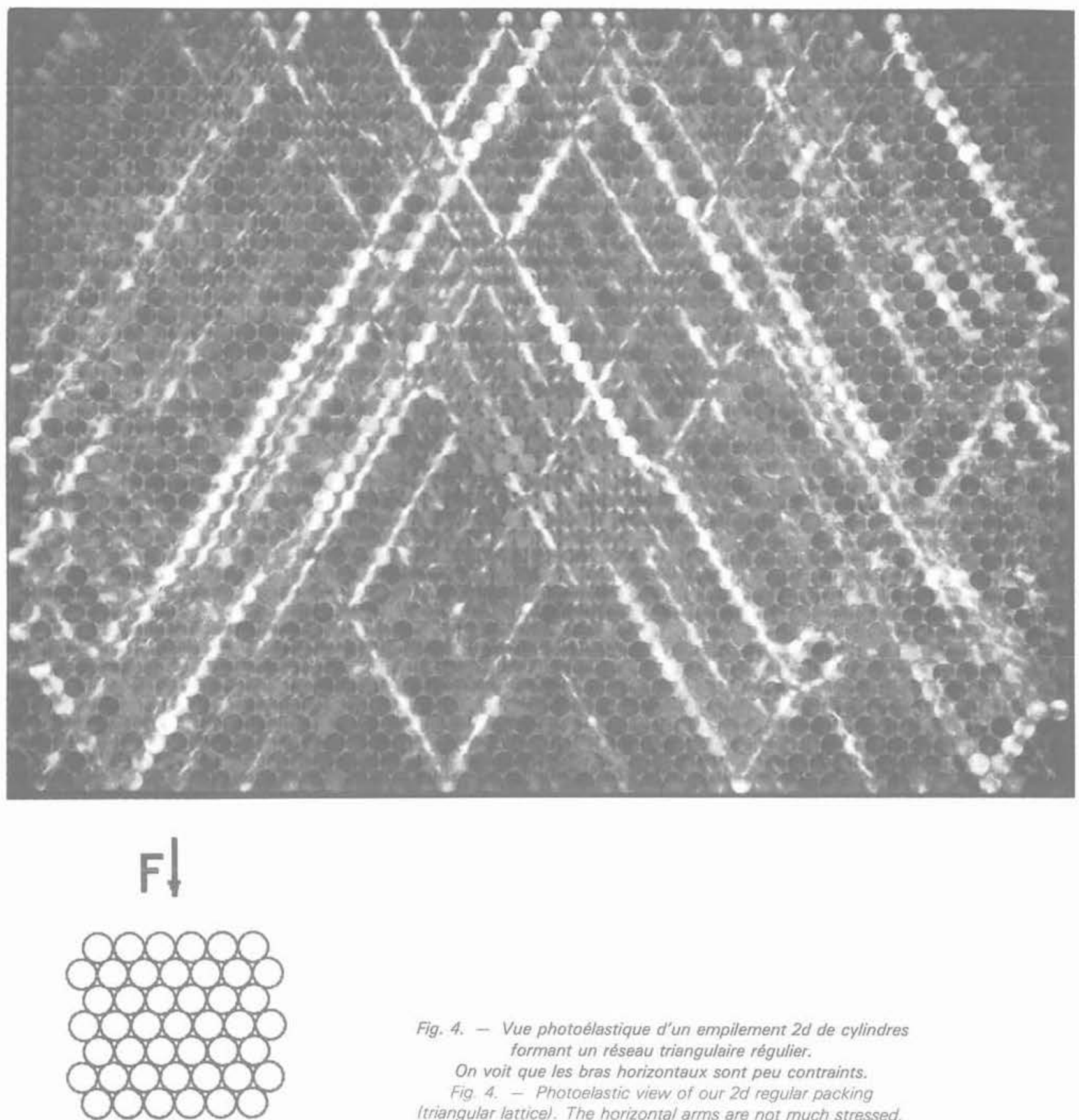

\author{
Fig. 4. - Vue photoélastique d'un empilement $2 d$ de cylindres \\ formant un réseau triangulaire régulier. \\ On voit que les bras horizontaux sont peu contraints. \\ Fig. 4. - Photoelastic view of our $2 d$ regular packing \\ (triangular lattice). The horizontal arms are not much stressed.
}

même si l'ordre classique de translation est détruit. L'ordre d'orientation (qui est dans ce cas défini à partir des directions des liens du réseau) peut en effet être préservé à l'échelle de l'échantillon, si les défauts de réseau (disinclinaisons et dislocations) ne sont pas trop nombreux (RUBINSTEIN et NELSON 1982) ; son rôle semble important pour les propriétés mécaniques, même dans les cas où il ne peut être défini qu'à courte distance comme dans les empilements désordonnés : il permet en effet de définir l'ordre de grandeur de la distance sur laquelle se font sentir les corrélations dans la transmission des forces.

Nous avons cherché à déterminer de façon plus quantitative l'importance du réseau des fortes contraintes. Le réseau étant ordonné, et le frottement entre grains n'étant pas négligeable, il est possible de créer des lacunes dans l'empilement, c'est-à-dire d'enlever des cylindres sans détruire l'équilibre des cylindres voisins. En nous appuyant sur les observations faites en photoélasticité, nous avons alors étudié la loi de compression en fonction de la concentration en lacunes, limitée à $5 \%$ (TRAVERS et al. 1987). Nous avons créé successivement trois sortes de lacunes :

1. des lacunes "éclairées »: les cylindres enlevés sont situés à un nceud du réseau des plus fortes contraintes. Ces lacunes sont distribuées de façon homogène dans l'échantillon.

2. des lacunes « sombres", également distribuées de 


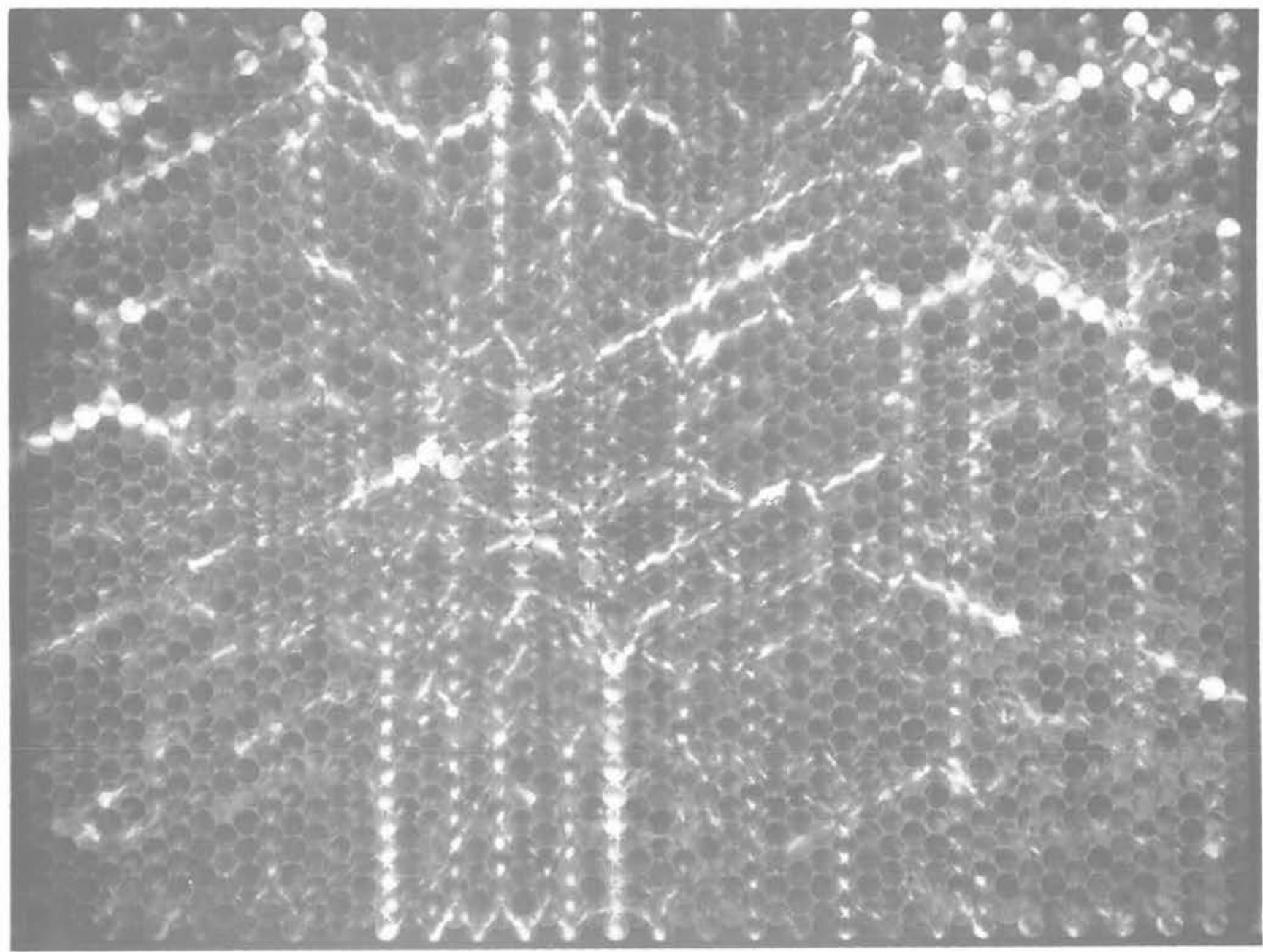

$\mathrm{Ft}$

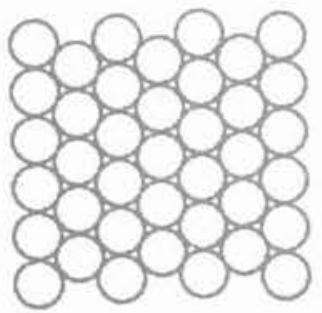

Fig. 5. - Vue photoélastique d'un empilement semblable d̀ celui de la figure 4, mais tourné de $90^{\circ}$.

La transmission des contraintes

par les bras verticaux est importante.

Fig. 5. - Photoelastic view of a packing similar to that on fig. 4 but turned by $90^{\circ}$. The transmission of the stresses

through the vertical arms is important.

façon homogène. Les cylindres correspondants n'interviennent que très peu dans la transmission des contraintes.

3. des lacunes " aléatoires " dont la position est déterminée à partir d'un générateur de nombres pseudoaléatoires.

En observation naturelle, les systèmes résultant de ces trois opérations paraissent géométriquement identiques. Cependant, leurs comportements mécaniques sont tout à fait différents comme le montre la figure 6 sur laquelle nous avons porté les variations de l'exposant $\mathrm{m}$ en fonction de la concentration $\mathrm{p}$ en lacunes.
Pour les lacunes * éclairées *, ces variations sont très importantes : pour $\mathrm{p}=0,05$, lexposant $\mathrm{m}$ a déjà chuté de $35 \%$; $m$ reste constant pour les lacunes * sombres * et les lacunes * aléatoires * donnent un comportement intermédiaire.

Ces expériences mettent en évidence l'importance de l'hétérogénéité de la distribution spatiale des contraintes intergranulaires. Le fait que les lacunes « sombres * n'aient aucune influence sur la réponse mécanique pour de petites déformations renforce le concept de « porosité mécanique » par opposition à la porosité géométrique : un rôle essentiel dans la déformation est joué par la fraction de grains relativement faible qui 
constitue le réseau des plus fortes contraintes.

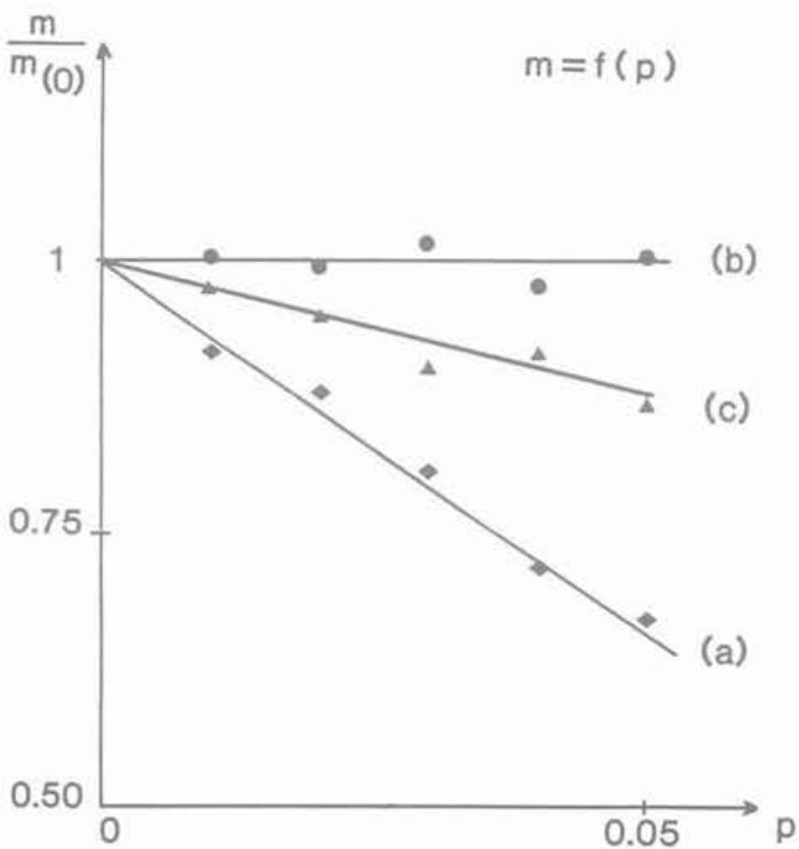

Fig. 6. - Variations de l'exposant macroscopique en fonction de la fraction de lacunes créees, dans les trois cas considérés dans le texte : a) lacunes " éclairées 》, b) lacunes " sombres", c) lacunes " aléatoires 1.

Fig. 6. - Variations of the macroscopic exponent as a function of the percentage of vacancies,

in the three cases considered in the text. a) « bright \# vacancies,

b) "dark y vacancies, of "random y vacancies.

\section{5. ÉTUDE DU DÉSORDRE DE COMPOSITION}

L'introduction de lacunes dans un empilement constitue une première étape vers le désordre de composition que nous allons envisager maintenant de façon plus générale en considérant le cas de mélanges aléatoires de cylindres de plexiglas (proportion p) et de cylindres de caoutchouc (proportion $1-p$ ) de même diamètre. Nous constituons ainsi un composite caractérisé par un grand contraste entre les propriétés mécaniques des deux phases. L'étude de ce type de système peut être abordée dans le cadre de la théorie de la percolation (de GENNES 1976) que nous décrivons brièvement avant de présenter nos résultats expérimentaux.

\subsection{La percolation}

La théorie de la percolation a été introduite pour décrire le passage d'un fluide dans un filtre partiellement obstrué. Elle a par la suite été appliquée à l'étude de nombreux problèmes concernant la matière en désordre, en particulier la conductivité électrique de mélanges (KIRKPATRICK 1973). Ses développements les plus récents portent sur les propriétés mécaniques des matériaux hétérogènes (GUYON et ROUX 1987).

Considêrons un mélange de deux types de grains de propriétés différentes, par exemple des grains conducteurs en concentration p et des grains isolants en concentration 1 - p. Pour de faibles valeurs de $p$, nous avons des amas finis de grains conducteurs isolés les uns des autres (figure 7a) : si deux électrodes sont placées sur les côtés supérieur et inférieur de l'échantillon, le mélange apparaît isolant. Lorsque $p$ atteint une valeur caractéristique $p_{c}$, appelée seuil de percolation. un "amas infini " connecté de grains conducteurs

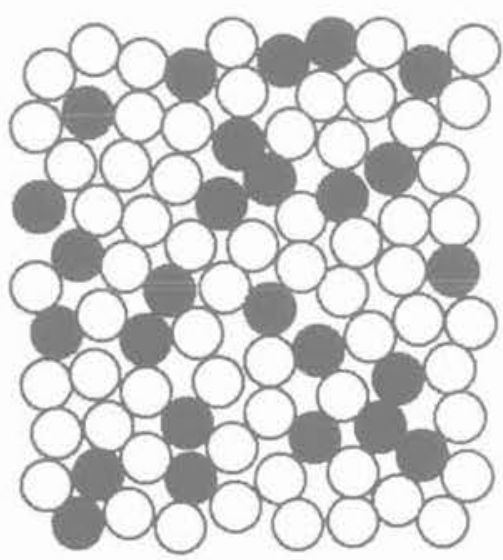

(a)

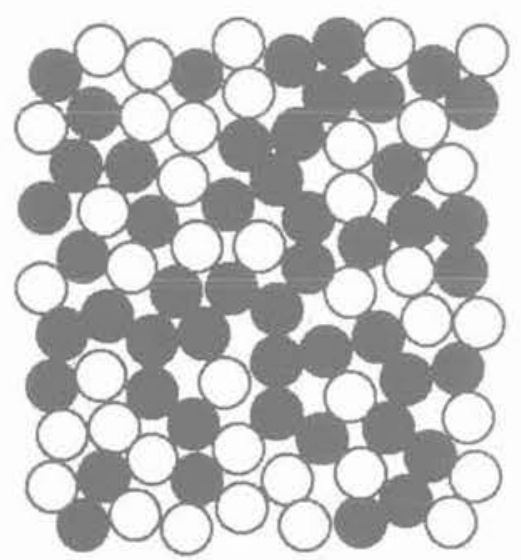

(b)

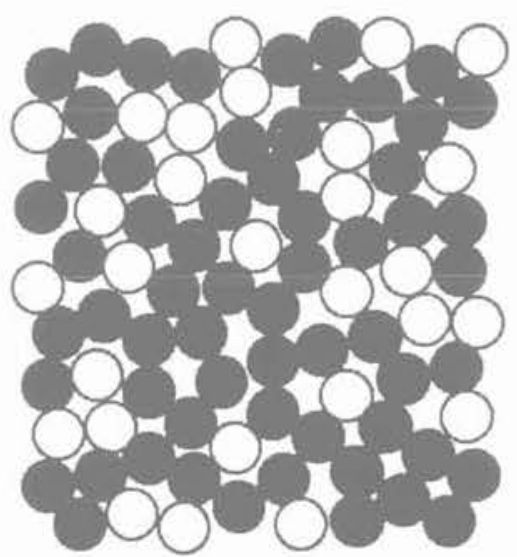

(c)
Fig. 7. - Mellanges de grains conducteurs (noirs) en proportion p et de grains isolants (blancs) en proportion 1-p :

a) pour une faible valeur de $p$,

b) légèrement au-dessus du seuil de percolation $p_{c}$ c) pour $p>p_{c}$
Fig. 7. - Mixtures of conducting (dark) and isulating (white) grains in percentage $p$ and $1-p$ respectively :

a) for small $\rho$, b) just above the percolation threshold $\rho_{c}$ c) for $p>p_{c}$ 
apparaît, entraînant une valeur non nulle de la conductivité du mélange. Une partie de l'amas infini contient des chaînons qui sont des impasses, les $\alpha$ bras morts , et qui sont donc inefficaces pour la conduction. L'amas infini débarrassé des bras morts est appelé squelette. La figure $7 \mathrm{~b}$ montre un échantillon dans lequel $\mathrm{p}$ est légèrement supérieur à $\mathrm{p}_{\mathrm{c}}$. Lorsque $\mathrm{p}$ augmente au-delà de $\mathrm{p}_{\mathrm{c}}$, l'amas infini se développe, absorbant progressivement tous les amas finis (figure 7c) jusqu'à occuper tout l'espace à $\mathrm{p}=1$.

Les notions d'amas infini et de seuil correspondent en fait à un échantillon de dimensions infinies. La valeur du seuil de percolation dépend de la géométrie du mélange considéré. A 2 dimensions, pour un mélange de grains cylindriques arrangés selon le réseau triangulaire régulier, $\mathrm{p}_{\mathrm{c}}=0,5$.

Considérons maintenant le même type de mélanges, mais formés de grains " durs " et de grains que nous supposons, pour simplifier, infiniment \& mous ». Dans ce cas particulier des milieux granulaires, la transmission des forces se fait par les contacts entre grains, et le modèle de percolation à force centrale (FENG et SEN 1984) semble particulièrement bien adapté. Au seuil de percolation $\mathrm{p}_{c}$, les grains durs forment un amas continu d'un bord à l'autre de l'échantillon, mais les déformations des bras du squelette pouvant se faire sans dépense d'énergie, le milieu ne présente aucune résistance à une sollicitation mécanique. Pour qu'il résiste à la contrainte extérieure, il est nécessaire d'augmenter la proportion de grains durs au-delà d'un seuil de rigidité $\mathrm{p}_{\mathrm{R}}$ pour lequel l'amas infini présente suffisamment de structures fermées pour s'opposer à la déformation angulaire (FENG et SEN 1984) ; dans cette structure rigide, les motifs triangulaires, à $2 \mathrm{~d}$, jouent un rôle primordial.

\subsection{Résultats expérimentaux et analyse}

Dans la zone de force intermédiaire, nous obtenons pour la relation contrainte-déformation une loi en puissance du type (2) pour toutes les compositions étudiées. Sur les figures $8 \mathrm{a}$ et $8 \mathrm{~b}$ sont portées les variations de l'exposant $m$ et du préfacteur $F_{0}$ en fonction de la concentration en cylindres de plexiglas. La courbe $\mathrm{Ln} \mathrm{F}_{\mathrm{o}}(\mathrm{p})$ montre une faible cassure au seuil de percolation $\mathrm{p}_{\mathrm{c}}=0,5$ et une cassure plus importante à $\mathrm{p}=$ 0,7 . Les variations de $\mathrm{m}(\mathrm{p})$ présentent également une cassure à $\mathrm{p}=0,7$.

Pour les faibles valeurs de p, ce sont essentiellement les propriétés du caoutchouc, modifiées par les inclusions de grains durs, qui déterminent les propriétés mécaniques de l'échantillon. L'amas " infini » qui apparaît au seuil de percolation $p_{s}=0,5$ n'est pas stable vis-à-vis de la contrainte appliquée ; il apporte sa contribution à la déformation par la flexion de ses éléments, squelette et bras morts. Ce n'est que lorsque la concentration en cylindres de plexiglas devient suffisante que l'amas infini devient mécaniquement stable. Ceci est produit vers $p=0,7$ qui apparaît donc comme un seuil de rigidité. Les études photoélastiques montrent qu'un réseau connecté des fortes contraintes n'existe que pour $p>0,7$. Ce résultat est compatible avec plusieurs simulations numériques récentes (LEMIEUX, BRETON et TREMBLAY 1985, STAUFFER, HERR MANN et ROUX 1987).
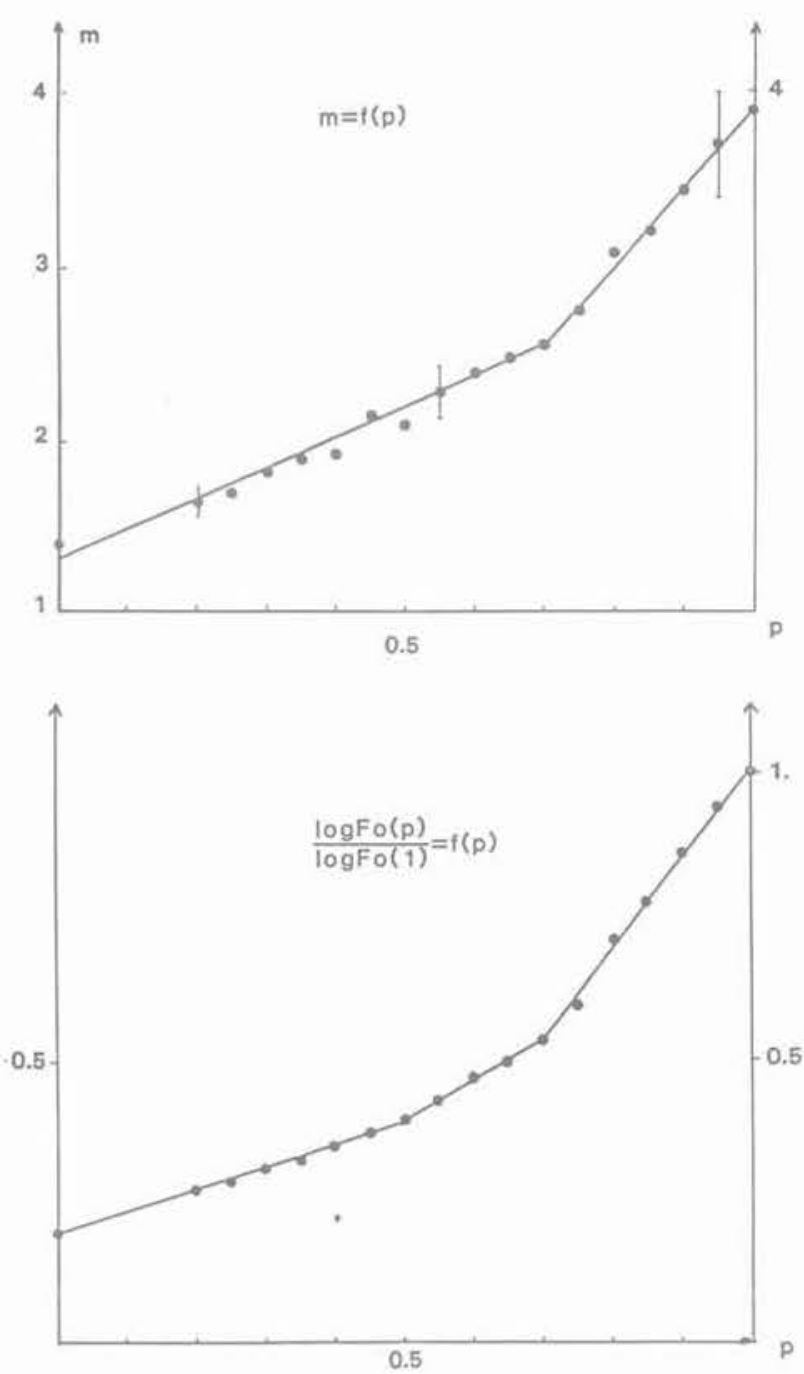

Fig. 8. - Variation avec la proportion p de cylindres de plexigias a- de l'exposant macroscopique $m$, b- du préfacteur $F_{o}$.

Fig. 8. - Variation with the percentage p of plexiglass cylinders a) of the macroscopic exponent $m$, b) of the prefactor $F_{0}$.

\section{INFLUENCE DES PAROIS EFFETS DE TAILLE}

Il est nécessaire de préciser à partir de quelle taille les empilements sont assez " grands " pour que les résultats obtenus au paragraphe précédent aient une portée générale. Les effets de taille purs sont modifiés par la présence des parois du récipient si bien qu'il est difficile de bien évaluer la part respective de ces facteurs.

Nous avons longuement décrit l'aspect purement géométrique de l'effet de paroi dans la section 3. Rappelons seulement que son incidence sur la géométrie de l'empilement est faible dès que les couches « périphériques" sont en nombre négligeable par rapport au nombre total. Nous avons également analysé l'effet de « paroi-piston » (section 4) et montré que le réseau des fortes contraintes n'est pas déterminé intrinsèquement 
par la paroi mobile. Dans ce paragraphe, nous nous intéressons donc surtout aux conséquences mécaniques de la variation de taille de l'échantillon.

Pour éviter d'inclure des effets supplémentaires, nous étudions ici des cylindres identiques, c'est-à-dire constitués du même matériau. Nous prenons à nouveau le. plexiglas, en raison de ses propriétés photoélastiques mais aussi à cause de la valeur élevée de l'exposant macroscopique mesuré. Dans cette série d'expériences, les cylindres présentent un meilleur état de surface, si bien que l'exposant $m$ est un peu moins élevé : $m=3,4 \pm 0,4$. Nous utilisons plusieurs approches différentes qui aboutissent à des conclusions similaires : il existe à grande et petite taille des comportements différents. Nous observons déjà un comportement * asymptotique * pour un échantillon $30 \times 30$ c'est-à-dire de taille bien inférieure à la taille maximale $44 \times 48$ de nos empilements.

\section{1. étude photoélastique}

L'étude photoélastique d'empilements de largeur $\mathrm{L}$ fixée ( $\mathrm{L}=44$ ou 45 cylindres) mais de hauteur $n$ variable ( $\mathrm{n}=$ nombre de couches ou rangées) montre l'existence de deux comportements distincts à petite et grande hauteurs, comme on peut le voir sur les photos de la figure 9. Quand l'échantillon comporte moins de 10 rangées, nous observons des bras isolés, fortement contraints, allant du haut en bas de l'empilement. Lorsque le nombre de rangées croît, le système se connecte et un réseau apparaît. Quand $n>20-24$ rangées, le réseau apparaît macroscopiquement homogène.

\section{2. influence des frottements sur les parois verticales}

Un problème posé par la compression $\alpha$ uniaxiale n des systèmes granulaires est l'estimation du rôle des frottements le long des parois verticales, qui peuvent modifier le comportement mécanique. DANTU (1967) a montré que lorsque la largeur est très petite devant la hauteur, la partie inférieure de l'échantillon peut " ignorer " la pression appliquée en haut; les grains profonds servent alors de points d'ancrage pour la création de voûtes. Si on admet que le coefficient de frottement sur la paroi est constant (ou plutôt que les effets des frottements sont les mêmes sur toute la hauteur de l'échantillon), la force verticale sur une rangée à la hauteur $z$ ( $z=0$ en haut de lempilement) varie comme $F(z)=F(0) e^{-\alpha z}$, résultat couramment admis pour les empilements. Cependant, les belles expériences de ITO et KUNO (1978) sur de plus petits échantillons (hauteur $\leqslant$ largeur) ont montré que les effets des frottements sont plus faibles dans la partie supérieure : ceci serait en majeure partie dú aux déplacements relatifs grains-parois importants en haut de l'échantillon, très faibles à la base (HARTMANN 1964).

Pour étudier l'effet des frottements sur les parois verticales, dans le cas où le rapport d'aspect (hauteur/largeur) est inférieur à 1 , nous avons mesuré, à l'aide de jauges de déformation préalablement étalonnées, la force transmise au bas de l'empilement pour différentes hauteurs n de celui-ci, la largeur étant maintenue constante (44-45 cylindres). Le rapport de la force transmise à la force appliquée est très proche de 1 pour
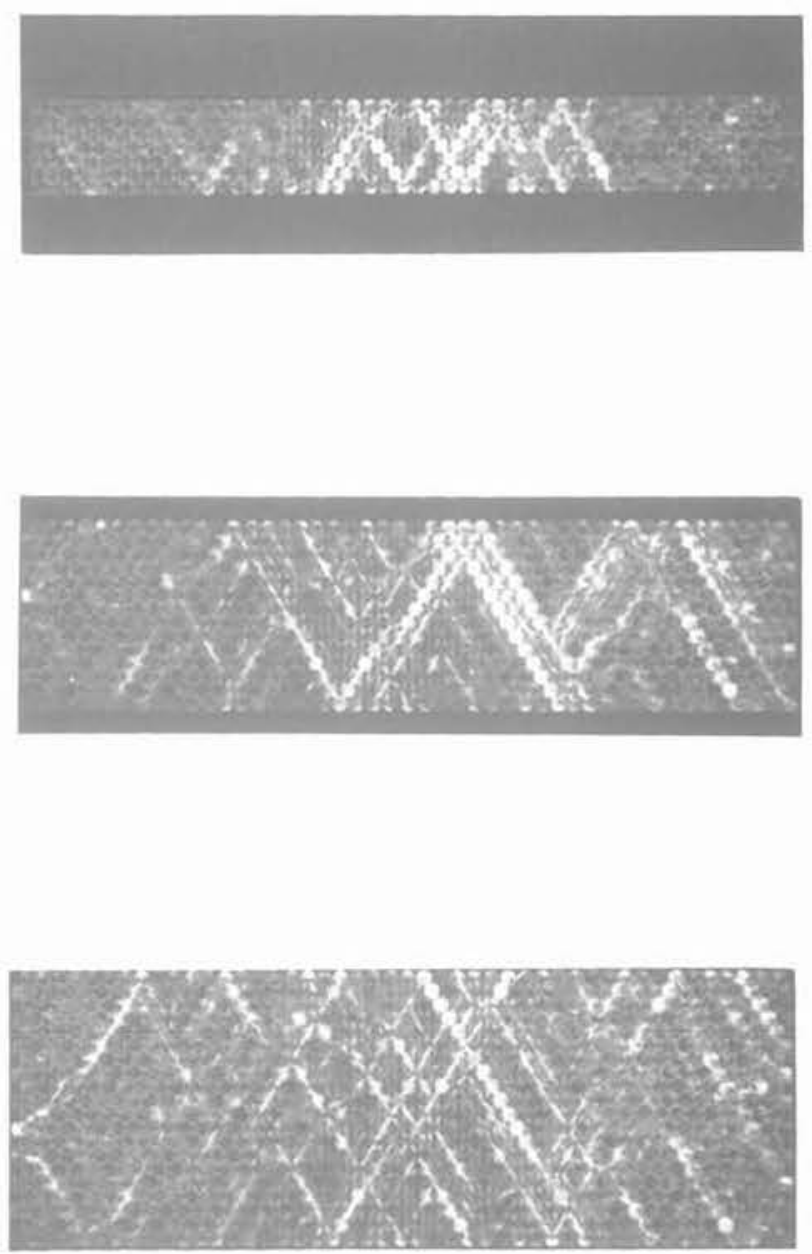

Fig. 9. - Visualisation des fortes contraintes à $F=2000 \mathrm{~N}$ pour des empilements réguliers de cylindres de plexiglas de différentes hauteurs:

a) $n=7$ (en dessous de la transition),

b) $n=14$ (dans la zone de transition),

c) $n=20$ (légèrement au-dessus de la transition).

Fig. 9 - Visualization of the strongest stresses network at

$F=2000 \mathrm{~N}$ for regular packings of plexiglass cylinders of different heights

a) $n=7$ (below the transition).

b) $n=14$ (transitional casel.

c) $n=20$ (just above the transition).

$\mathrm{n}<10$, il évolue pour $10<\mathrm{n}<20$ et se stabilise à une valeur voisine de 0,92 pour $n>20$ (figure 10). Ceci montre que dans notre cas les frottements sur les parois verticales sont faibles ce qui est compatible avec les observations photoélastiques et avec les expériences à petite hauteur de ITO et KUNO (1978).

Par ailleurs, nous avons mesuré, sur un empilement de grande taille ( 48 rangées de 44 ou 45 cylindres), la force résultante normale aux parois verticales. Cette force varie linéairement avec la force appliquée lorsque celle-ci augmente, le rapport des deux étant de l'ordre de $1 / 4$. Le coefficient de pression latérale $K_{0}$ est donc constant. 


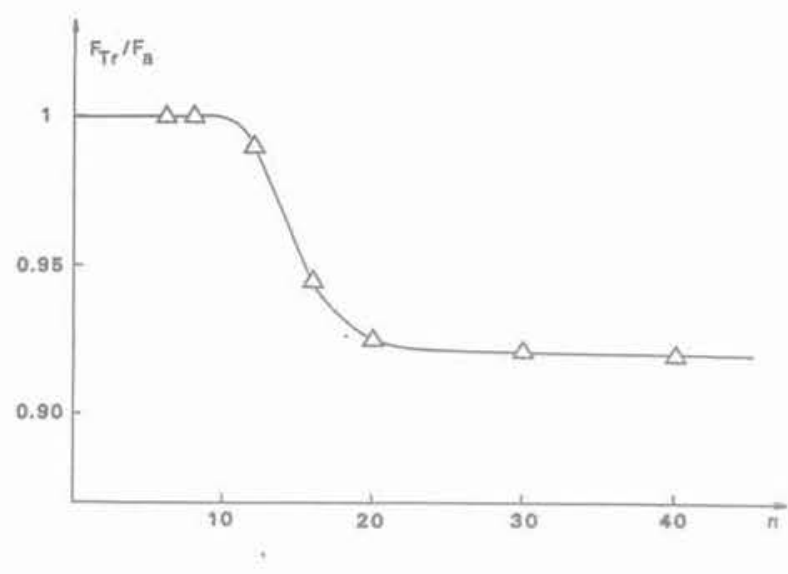

Fig. 10. - Variation du rapport force transmise $\left(F_{T}\right) /$ force appliquée $\left(F_{3}\right)$ pour différentes hauteurs $n$.

Fig. 10. - Variations of the ratio transmitted force to applied force $\left(=F_{T r} / F_{j}\right.$ for $F_{a}=2000 \mathrm{~N}$ and different heights $n$.

\subsection{Loi force-déformation et effets de hauteur}

Les valeurs expérimentales données ci-dessous sont obtenues à force croissante, au troisième cycle de pression et sont la moyenne sur trois empilements différents de mêmes dimensions. Nous avons étudié des échantillons dont le nombre de rangées horizontales varie de $n=6$ à $n=44$ avec alternativement 44 et 45 cylindres par rangée. La gamme de force est
$0-2000 \mathrm{~N}$. Nous mesurons la déformation verticale $\Delta \mathrm{h}$ de l'échantillon en fonction de la force appliquée $\mathrm{F}$.

Les tracés en échelle log-log de $F=g(\Delta h)$ montrent deux parties linéaires : il y a un comportement à " faible force " (phase de consolidation) et un comportement à "force élevée » (phase consolidée), qui peuvent être décrits par une loi en puissance. La force de transition $F_{\mathrm{T}}$, à la cassure entre les deux régimes, est pratiquement constante et proche de $600 \mathrm{~N}$ quand $n<20$, puis change rapidement pour une nouvelle valeur de $300 \mathrm{~N}$ quand $n>20$ (figure 11).

Pour une hauteur donnée, nous avons deux exposants macroscopiques, $\mathrm{m}_{\mathrm{b}}$ à faible force et $\mathrm{m}_{\mathrm{h}}$ dans la zone consolidée ; $\mathrm{m}_{\mathrm{b}}$ est constant et voisin de 1,5 quand $\mathrm{n}<10$, il augmente pour $10<\mathrm{n}<20$, puis redevient constant et de l'ordre de 2 pour $n>20 ; m_{h}$, qui est très grand - de l'ordre de 10 - pour des petites valeurs de $n$ (une loi en puissance a-t-elle encore un sens ?), décroît et devient pratiquement constant et égal à $3,4 \pm 0,4$ pour $n>24$, sans qu'il soit cependant possible d'exclure un comportement transitionnel à variation lente (figure 12). A partir des mêmes expériences, nous avons étudié aussi le taux de déformation par rangée $\Delta h / n$ en fonction du nombre de rangées. Il est constant pour $n>20$ (figure 13). Ceci est compatible avec une description théorique dans laquelle toutes les rangées se comporteraient de la même façon (KO et SCOTT 1967).
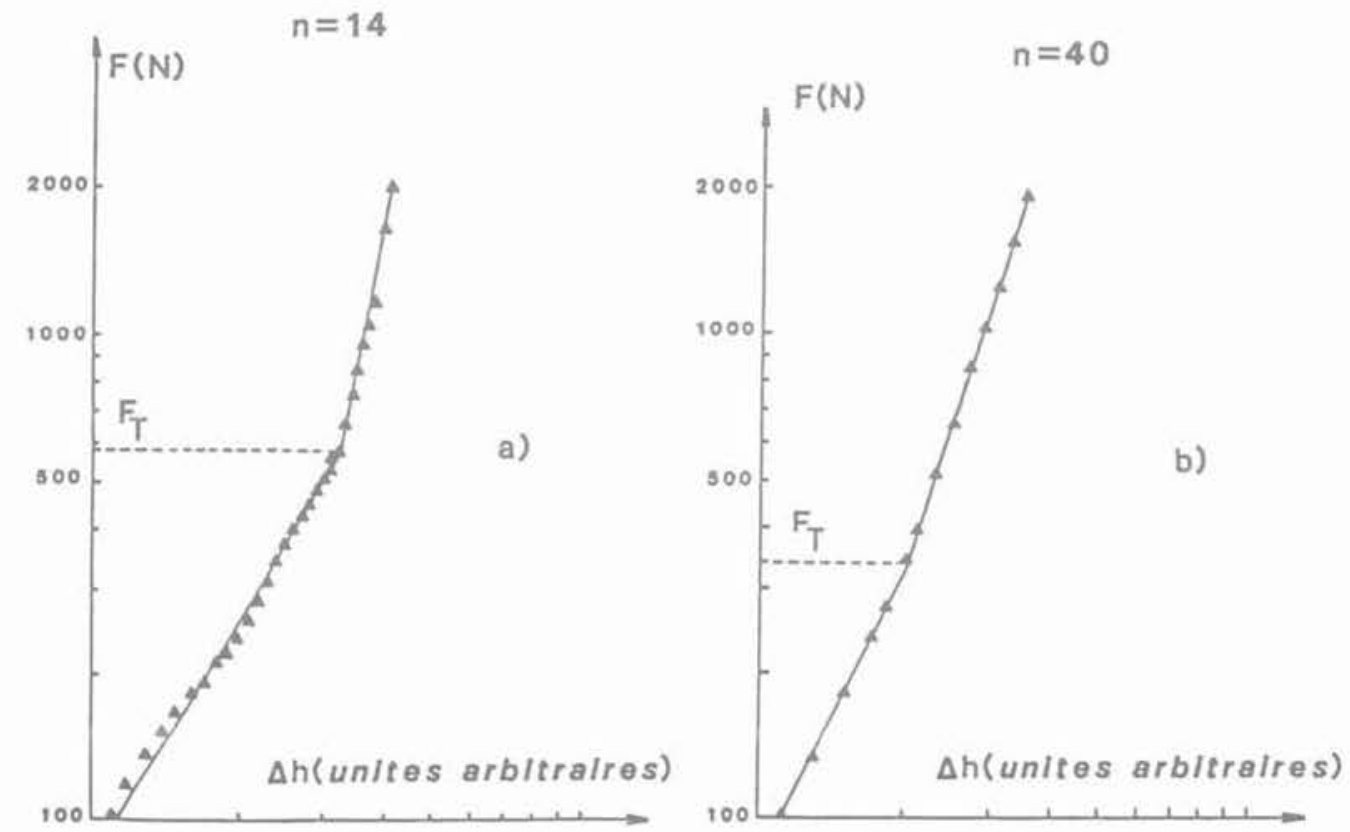

Fig. 11. - Loi force-déformation en échelle log-log

a) à faible hauteur $(n=14)$,

b) à grande hauteur $(n=40$ )

Fig. 11. - Stress-strain law in log-log scale .

a) for small height $(n=14)$,

b) for large height in $=40$ ). 


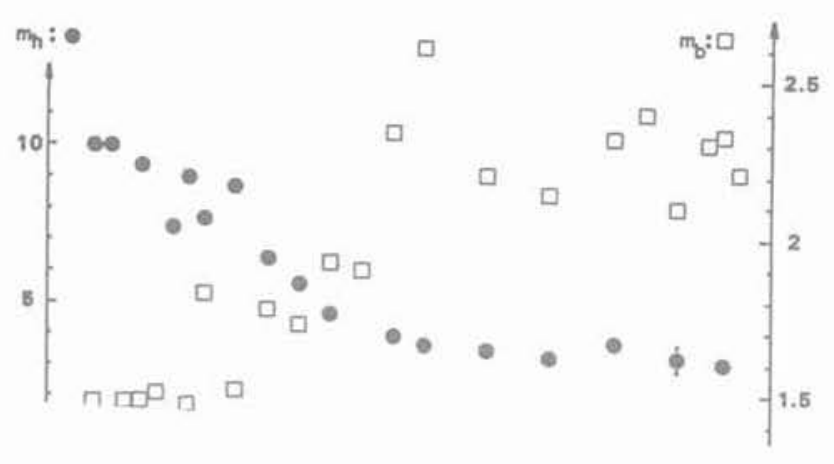

Fig. 12. - Variations des exposants $m_{h}$ et $m_{b}$ avec la hauteur $n$. Deux régimes apparaissent nettement.

Fig. 12. - Exponents $m_{h}$ and $m_{b}$ as a function of the height $n$ of the packing. The two regimes appear clearly as $n$ increases.

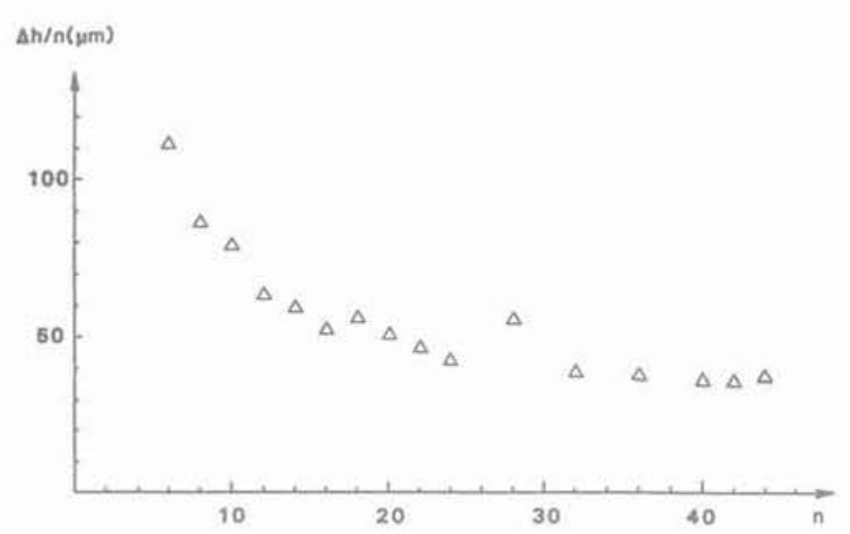

Fig. 13. - Taux de compression $\Delta h / n$ en fonction de la hauteur $n$.

Fig. 13. - Compression rate $\Delta h / n$ as a function of height $n$.

\section{4. influence de la largeur}

Nous avons effectué la même étude en faisant varier la largeur L de l'empilement pour une hauteur constante $\mathrm{n}=46$. La largeur est modifiée par l'addition de cales rectifiées. Une légère incommensurabilité entre la largeur de l'empilement et la taille du montage n'altère pas les exposants de la loi en puissance ; cependant, elle peut modifier la hauteur de l'échantillon en permettant une meilleure restructuration *latérale ». D'autre part, nous travaillons ici dans une gamme de pression constante, le maximum de pression correspondant au maximum de force dans les expériences précédentes. Nous observons à nouveau deux régimes : un à " basse pression ", l'autre à " haute pression ». En dehors des erreurs expérimentales, qui peuvent être plus importantes ici, la pression de transition $\mathrm{P}_{\mathrm{T}}$, définie comme la force de transition $\mathrm{F}_{\mathrm{T}}$, ne change pas pour $\mathrm{n}=46$ quand la largeur $\mathrm{L}$ varie de $\mathrm{L}=6$ à $\mathrm{L}=46$.

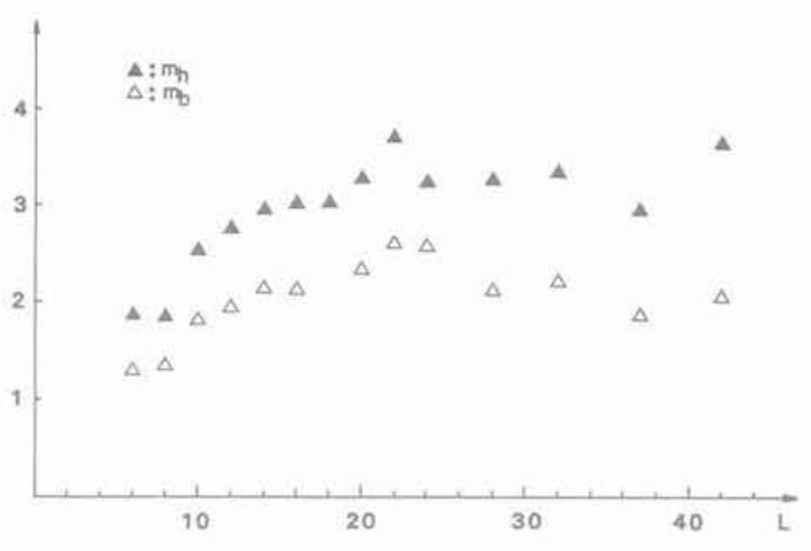

Fig. 14. - Variations des exposants $m_{h}$ et $m_{b}$ avec la largeur $L$ pour un empilement de 46 couches.

Fig. 14. - Exponents $m_{h}$ and $m_{b}$ as a function of the width $L$ of the packing for a constant height $n=46$.

L'évolution des exposants $m_{b}$ et $m_{h}$ avec la largeur est représentée sur la figure 14 . Un régime transitoire pour $10<\mathrm{L}<20$ apparait clairement. L'exposant $\mathrm{m}_{\mathrm{b}}$ est proche de 1,5 pour $\mathrm{L}<10$, il augmente dans la zone $10<\mathrm{L}<20$, puis devient constant et de l'ordre de 2 pour $\mathrm{L}>20$. L'exposant supérieur $\mathrm{m}_{\mathrm{h}}$ est constant et voisin de 1,8 quand $\mathrm{L}<10$, puis augmente dans la zone $10<\mathrm{L}<20$, et devient égal à la valeur asymptotique $m_{h}=3,4$ quand $L>20$.

Une étude identique a été faite dans une situation opposée, c'est-à-dire pour une petite hauteur $n=8$; les fluctuations sont si importantes que nous ne pouvons qu'indiquer une tendance : il semble que pour $\mathrm{L}$ élevé $\mathrm{m}_{\mathrm{b}}$ soit plus ou moins constant, de l'ordre de 5 , cependant que $\mathrm{m}_{\mathrm{b}}$, comme dans le premier cas, croît vers $2-2,5$.

Cette étude donne des résultats très similaires à ceux obtenus précédemment : il y a deux régimes suivant la largeur de l'échantillon. Les zones de transition pour $10<\mathrm{L}<20$ et $10<\mathrm{n}<20$ sont tout à fait comparables. Les limites de ces régimes $(L \sim 10 ; L \sim 20)$ sont compatibles avec la longueur définie à partir des lignes connectées observées en photoélasticité comme nous le verrons plus loin.

Pour compléter cette étude, nous avons fait des mesures quand la hauteur $n$ et la largeur L varient simultanément, leur rapport $n / L$ restant égal à 1 . Nous en tirons les mêmes conclusions : l'exposant supérieur décroît depuis $m_{h} \geqslant 5$ pour $n=L=20$ jusqu'à sa valeur asymptotique 3,4 quand nous avons un empilement $40 \times 40$.

\section{CONCLUSION}

En faisant appel à plusieurs types d'expériences, nous avons étudié la compression cedométrique de milieux granulaires $2 \mathrm{~d}$. Ces expériences montrent deux régimes suivant la force appliquée : un régime de consoli. dation, et un régime consolidé ; dans chacun de ces 
régimes, la relation entre la force macroscopique et la déformation $\Delta h$ peut être exprimée par une loi en puissance. Dans ces deux régimes également, le comportement observé dépend de la taille de l'échantillon. Il apparait que, pour l'essentiel, le comportement observé est lié au désordre géométrique (pourtant faible) présenté par nos empilements.

En effet, nos empilements, avant la première application de la force, présentent un désordre et une anisotropie dans la répartition des contacts, l'anisotropie étant encore accentuée après plusieurs cycles de pression. La probabilité p pour un lien (contact géométri. que entre 2 grains) d'exister est très grande, même avant le troisième cycle de pression et elle est supé. rieure à 0,75 qui est sa valeur avant le premier cycle. Le long de chaque direction $Y$ et $Z$ (figure 2), les chaînons de longueur $s$ (formés de $s+1$ sites en contact) existent avec une probabilité $\mathrm{p}^{\mathrm{s}}(1-\mathrm{p})$, leur longueur moyenne étant $\langle s\rangle=p /(1-p)$. Ces chaînons continus seraient le point de départ des bras rectilignes rigides, visualisés en photoélasticité pour $n<10$; ils ne seraient pas assez longs pour relier le piston supérieur à la paroi inférieure horizontale quand $n>20$ : la structure rigide (ou stable) prendrait alors la forme du réseau des fortes contraintes observé en photoélasticité pour les grands échantillons. La longueur caractéristique de nos empilements pour ce problème mécanique serait alors cette longueur de stabilité d'un bras rigide.

Toutefois l'absence de contacts, et les défauts géométriques de nos cylindres, font que l'empilement n'est pas rigide à force faible ; il peut être déformé à cause de glissements quasi réversibles. Quand l'échantillon est comprimé, il se crée progressivement des contacts actifs (ou rigides) qui à leur tour génèrent des amas rigides, qui sont seulement statistiques puisque un petit incrément de force peut modifier le réseau de plus fortes contraintes (voir par exemple le film réalisé par le $\mathrm{LCPC}^{*}$ ). Ces amas rigides grandissent afin de former une structure stable allant du haut en bas de l'échantillon qui se trouve ainsi consolidé. Ceci ressemble au processus de percolation décrit dans la section 5. La force nécessaire pour obtenir ces amas rigides correspondrait alors à la force de transition $\vec{F}_{T}$ que nous avons observée entre les deux régimes mécaniques. Cette force de transition dépend de la structure, du coefficient de frottement entre les grains, des caractéristiques mécaniques intrinsèques et des défauts géométriques du matériau (distribution de taille, flèche de l'axe de nos cylindres de plexiglas, etc.) et plus globalement de l'histoire de l'échantillon ; par exemple, dans les empilements de cylindres d'acier, dont la définition géométrique est dix fois meilleure que celle des cylindres de plexiglas, et qui sont plus rectilignes, nous trouvons une force de transition bien plus basse. De même les deux valeurs de $F_{\mathrm{T}}$ à grande et petite hauteurs s'expliquent par des structures gêométriques différentes, soit en raison du rôle du piston (près de la paroi supérieure, le désordre est plus important) soit à cause des frottements sur les parois verticales qui sont différents dans les deux régimes, comme nous l'avons vu auparavant.

Ainsi, l'existence des deux régimes à basse force et dans la zone intermédiaire, semblables aux régimes obtenus dans les simulations numériques sur des réseaux de diodes Zener (ROUX et HERRMANN 1987) peut être expliquée d'un point de vue " percolation , en partant des contacts actifs. Il apparaît que l'influence de la hauteur peut être interprétée d'un point de vue géométrique et mécanique (principalement à cause des frottements sur les bords du montage). L'étude de l'influence de la largeur indique clairement que les effets géométrique et mécanique des parois ne sont pas des contributions importantes aux effets de taille. On aurait observé une évolution douce des paramètres au lieu de changements plus brutaux du type « saut *. L'interprétation de type statistique semble donc la mieux étayée expérimentalement ; elle est aussi très bien justifiée au niveau théorique.

Nos expériences ont souligné le rôle de la longueur caractéristique, plus grande que la taille des grains et définie à partir des propriétés géométriques des empilements. Cette longueur caractéristique est très sensible aux hétérogénéités, et probablement à l'ordre d'orientation. Nous pensons que beaucoup de points restent encore à śclaircir dans cette optique, et que des expériences sur des empilements $2 \mathrm{~d}$ et $3 \mathrm{~d}$ désordonnés restent à faire.

\section{BIBLIOGRAPHIE}

BEN AIM R. and LE GOFF P. (1968), La coordinance des empilements désordonnés de sphères, Powder Technology, 2, 1-12.

BIAREZ J. (1962), Contribution à l'étude des propriétés mécaniques des sols et de matériaux pulvérulents. Thèse d'État, Grenoble.

BIDEAU D. (1983), Relation entre les propriétés de transport de matériaux constitués d'empilements désordonnés isotropes et leurs caractéristiques géomé. triques, Thèse d'État, Rennes.

DANTU P. (1957), Contribution à l'étude mécanique et géométrique des milieux pulvérulents, août 1957 . Proceedings of the 4th International Conference on Soil Mechanics and Foundations Engineering, Londres.

DANTU P. (1967), Étude expérimentale d'un milieu pulvérulent compris entre deux plans verticaux et parallèles, Annales des Ponts et Chaussées IV.

GENNES (de) P.G. (1976), La percolation : un concept unificateur, La Recherche, 7, 919-927.

DRESCHER A. et de JOSSELIN de JONG G. (1972), Photoelastic verification of a mechanical model for the flow of a granular material, Journal of Mechanical Physics of Solids, 20, 337-351

FEDA J. (1982), Mechanics of particulate Materials, Elsevier Amsterdam.

FENG S, et SEN P.N. (1984), Percolation on elastic networks: new exponent and threshold, Physical Review Letters, 52, 216-219.

\footnotetext{
On peut se procurer ce film au Laboratoire Central des Ponts et Chaussées, 58, boulevard Lefebure, 75015 Paris.
} 
GAUTHIER C., BORGOTTI J.C. et SADOUDI A. (1987), Sur les distributions des temps de séjour d'un fluide dans des mélanges binaires de billes de verre: importance de la mise en place, Comptes rendus de l'Académie des Sciences de Paris. Soumis.

GUYON E. et ROUX S. (1987), Les matériaux hétérogènes, La Recherche, 18, n 191, 1050-1058.

HARTMANN H.S. (1964), Packing and compaction in dies of spherical particles, PhD Thesis MIT.

ITO Y. et KUNO H. (1978), An application of scattered-light photoelasticity in the determination of the side pressure distribution of the pressed powder bed, Powder Technology, 20, 127-131.

KIRKPATRICK S. (1973), Percolation and conduction, Review of Modern Physics, 45, 574-588.

KO H.Y. et SCOTT R.F. (1967), Deformation of sand in hydrostatic compression, Journal of the Soil Mechanics and Foundation Division, SM3, 137-156.

LEMIEUX M.A., BRETON P. et TREMBLAY A.-M.S. (1985), Unified approach to numerical matrix methods for disordered systems : applications to mixed crystals and to elasticity percolation, Le Journal de PhysiqueLettres, 46, Li-L7.

ROUX S. et HERRMANN H.J. (1987), Disorder induced non-linear conductivity, Europhysics Letters, 4, 1227-1231.

RUBINSTEIN M. et NELSON D. (1982), Order and deterministic chaos in hard-disk arrays, Physical Review, B, 26 6254-6275.

SCHNEEBELI G. (1956), Une analogie mécanique pour les terres sans cohésion, Comptes rendus de l'Académie des Sciences de Paris, 243, 125-126.

STAUFFER D., HERRMANN H.J. et ROUX S. (1987), Simulation of disordered systems of cylinders, Journal de Physique, 48, 347-52.

TRAVERS T., BIDEAU D., GERVOIS A., TROADEC J.P. et MESSAGER J.C. (1986), Uniaxial compression effects on $2 d$ mixtures of hard and soft cylinders, Journal of Physics, A 19 L, 1033-1038.

TRAVERS T., AMMI M., BIDEAU D., GERVOIS A. MESSAGER J.C. et TROADEC J.P. (1987), Uniaxial compression of $2 d$ packings of cylinders. Effects of weak disorder, Europhysics Letters, 4, 329-332. 